\title{
Comparison on Hysteresis Movement in Accordance with the Frictional Coefficient and Initial Angle of Clutch Diaphragm Spring
}

\author{
Hyun-sik Kim ${ }^{1,}$, Sung-mo Yang ${ }^{1, b}$ and Hyang-woo Kim ${ }^{2, \mathrm{c}}$ \\ ${ }^{1}$ Department of Mechanical System Engineering, Chonbuk National University, \\ Jeonju, 54896, South Korea \\ ${ }^{2}$ Department of Automotive Plant Industrial Engineering, Hanyeong College, \\ Yeosu, 59720, South Korea \\ ahyunsiclove@jbnu.ac.kr, ${ }^{b}$ yangsm@jbnu.ac.kr, chwkim4035@hanmail.net
}

Keywords: Hysteresis, Clutch diaphragm spring, Frictional coefficient, Initial angle.

\begin{abstract}
Clutch system influences the clutch pedal force of an operator in accordance with the characteristics of each part. Hence, the purposes of this study are to establish a mathematical model on the diaphragm spring of clutch cover and to identify the basic characteristics for each mathematical model. Also in this study, the characteristics of hysteresis as to the load-displacement will be analyzed depending on the initial angle and frictional coefficient. As a result of the interpretation, both the clamp load and release load tended to widen the hysteresis width when the frictional coefficient increased. This result implies that the load will also increase when the frictional force increases. Moreover, the hysteresis value has also increased when the initial pressure angle increased with uniform frictional coefficient. This result may be explained by the fact that higher hydraulic pressure of master cylinder was exerted to clutch system when a driver presses a pedal with a large angle. Comparing with the measured data, a frictional coefficient of 0.4 and an angle of 13 degrees would be considered most suitable.
\end{abstract}

\section{Introduction}

In a manual transmission vehicle, clutch is the shaft-splicing apparatus that can disconnect the power train when passing the rotation movement of a shaft to another shaft. It can stop the driven shaft without stopping the movement of the main shaft connected to the motor. Hence, it is widely used in a speed controller. In particular, it is often used in the automotive field. It is an essential device for a power system for transmission.

As for small and medium sized commercial vehicles in the automotive field, manual clutch is generally used. Given the transfer torque is large a hydraulic pressure device is additionally installed and used on the path where a clutch pedal is connected to a clutch. Such auxiliary device causes a change as to the characteristics of clutch operation. Moreover, they have the non-linear movement mechanism characteristics [1].
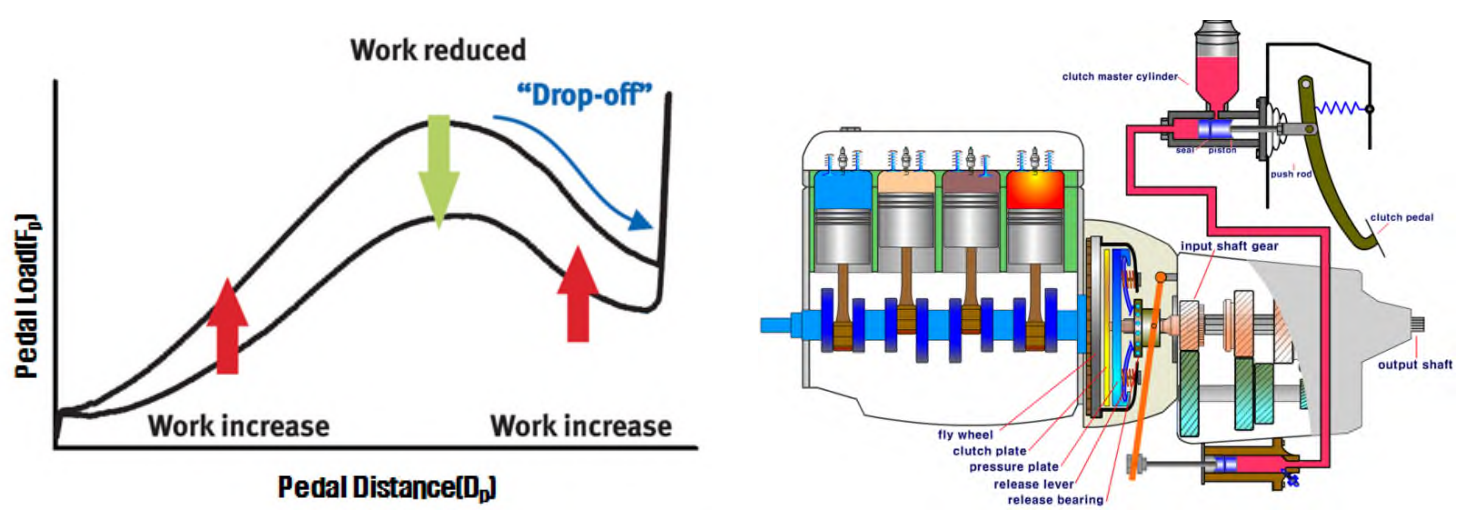

Fig. 1 Clutch system and hysteresis loop. 
The clutch system consists of the following components as shown in Fig. 1 [1]. They influence the clutch pedal force of a driver depending on the characteristics of each part. Therefore, this study aims to establish a mathematic model as to the diaphragm spring of clutch cover, identify the basic characteristics of each component and analyze the characteristics of hysteresis as to the load-displacement in accordance with the initial angle and frictional coefficient.

\section{Theoretical Background}

The components of a clutch system are as follows: clutch pedal, master cylinder, oil line, clutch booster, release fork, release bearing and clutch cover. The clutch operation system of a conventional commercial vehicle consists of the structure that operates the pressure plate of clutch cover over release fork and diaphragm spring after operating a clutch booster with the movement of fluid created in a master cylinder as shown in Fig. 2 [2].

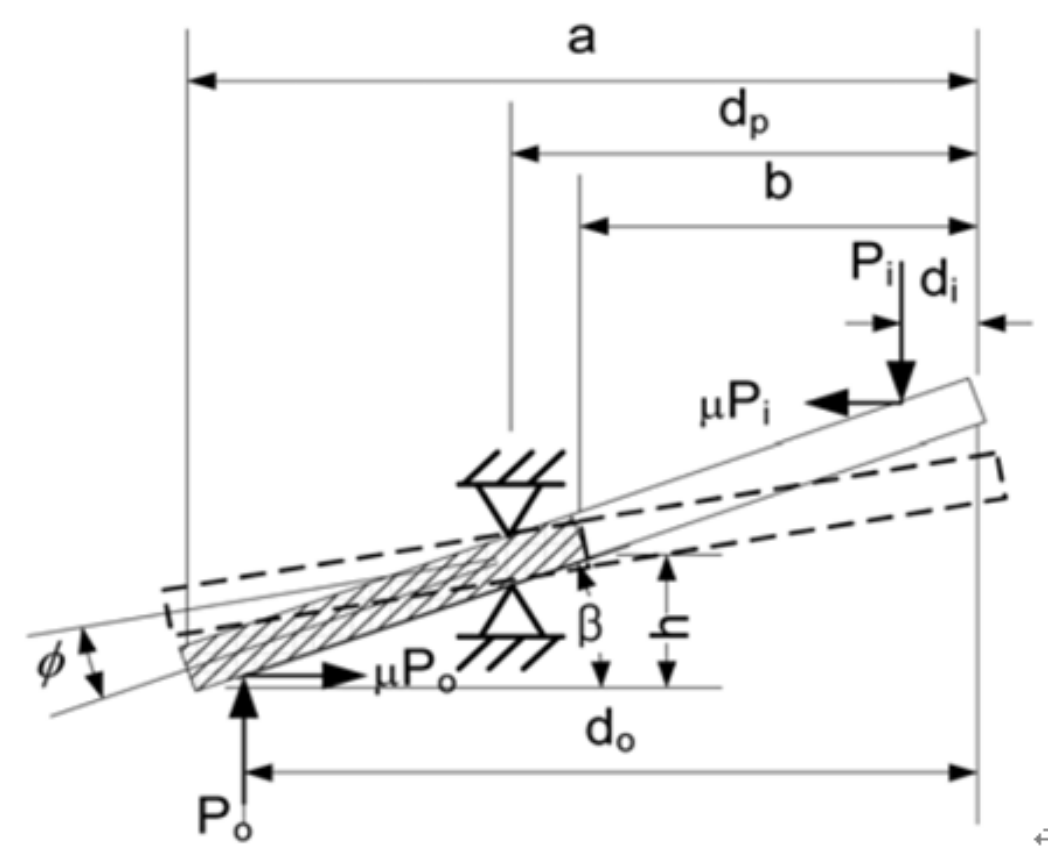

Fig. 2 Schematic of a diaphragm spring in deformation around fixed support or pivot.

A clutch can start an engine with no-load rotation without transmitting the power of an engine to the transmission. It has the function of transmitting to an adequate gear depending on the driving state in case of no-load state. In regard to the diaphragm spring of clutch cover to be applied in this study, it is possible to obtain the moment for a small portion in the lateral direction by calculating the moment by frictional force. Frictional force occurs around release bearing and pressure plate. The moment at this time is as follows $[2,3]$.

$$
\begin{aligned}
& d M_{i}= \pm \frac{\mu P_{i}}{2 \pi d_{i}} d_{i} d \theta\left[\left|\left(d_{p}-d_{i}\right) \tan (\beta-\varphi)\right|+\frac{t}{2} \cos (\beta-\varphi)\right] \\
& d M_{o}= \pm \frac{\mu P_{o}}{2 \pi d_{o}} d_{o} d \theta\left[\left|\left(d_{o}-d_{p}\right) \tan (\beta-\varphi)\right|+\frac{t}{2} \cos (\beta-\varphi)\right]
\end{aligned}
$$

Under the assumption that the moment by frictional force has a small degree of transformation, $d M_{i}, d M_{o}$ can be expressed in the following formula with $\cos (\beta-\varphi) \approx 1, \tan (\beta-\varphi) \approx \beta-\varphi$.

$$
\begin{aligned}
& d M_{i}^{*}= \pm \frac{\mu P_{i}}{2 \pi d_{i}} d_{i} d \theta\left[\left|\left(d_{p}-d_{i}\right)(\beta-\varphi)\right|+\frac{t}{2}\right] \\
& d M_{o}^{*}= \pm \frac{\mu P_{o}}{2 \pi d_{o}} d_{o} d \theta\left[\left|\left(d_{o}-d_{p}\right)(\beta-\varphi)\right|+\frac{t}{2}\right.
\end{aligned}
$$

The formula of moment as to release load and clamping load is as follows. 


$$
\begin{aligned}
& d M_{i}=\frac{\mu P_{i}}{2 \pi d_{i}} d_{i} d \theta\left[d_{p}-d_{i}-\frac{t}{2} \sin (\beta-\varphi)\right] \approx \frac{P_{i}}{2 \pi} d \theta\left(d_{p}-d_{i}\right) \\
& d M_{o}=\frac{\mu P_{o}}{2 \pi d_{o}} d_{o} d \theta\left[d_{o}-d_{p}-\frac{t}{2} \sin (\beta-\varphi)\right] \approx \frac{P_{o}}{2 \pi} d \theta\left(d_{o}-d_{p}\right)
\end{aligned}
$$

The moment of radial direction transformation and change in curvature for finger-type semi spring is as shown in the following formula.

$$
\begin{aligned}
& \mathrm{d} M^{\prime}=E t d \theta \varphi(\beta-\varphi)\left(\beta-\frac{\varphi}{2}\right)\left[\frac{1}{2}\left(a^{2}-b^{2}\right)-\frac{\left(a^{2}-b^{2}\right)}{\ln a-\ln b}\right] \\
& \mathrm{d} M^{\prime \prime}=\frac{E t^{3} \varphi d \theta}{12} \ln \frac{a}{b}
\end{aligned}
$$

This basic moment is parallel to the moment by external force. Therefore, it can be expressed in the following equilibrium equation.

$$
\mathrm{d} M_{i}+d M_{o}=d M_{i}^{*}+d M_{o}^{*}+d M_{i}^{\prime}+d M_{o}^{\prime \prime}
$$

The finger tip of diaphragm spring by release load $P_{i}$, that is, the displacement of release bearing part is identical to the displacement that combines the displacement by each displacement of conical spring, displacement of finger part by each displacement and deflection of finger by release load. Once the clamping load $P_{o}$ as to a given displacement or angle is determined, the final moment equilibrium equation for obtaining release load $P_{i}$ is as follows [3].

$$
P_{i}=\frac{P_{o}\left[\sigma \mu\left\{\left|\left(d_{o}-d_{i}\right)(\beta-\varphi)\right|+\frac{t}{2}\right\}-\left(d_{o}-d_{p}\right)\right]}{d_{p}-d_{i}-\sigma \mu\left\{\left|\left(d_{p}-d_{i}\right)(\beta-\varphi)\right|+\frac{t}{2}\right\}}
$$

Hence, it is possible to analyze the characteristics as to reaction force of diaphragm spring in relation to a clutch system.

\section{Simulation in Accordance with the Change in Frictional Coefficients}

It is possible to find a variable that corresponds to frictional coefficient $\mu$ of diaphragm spring by comparing the measured data of diaphragm spring. It can be done as follows with the measured result data.

Table 1. Parameter values of diaphragm spring.

\begin{tabular}{|l|l|}
\hline Parameter & Value \\
\hline $\mathrm{a}, \mathrm{b}$ & $159 \mathrm{~mm}, 130.5 \mathrm{~mm}$ \\
\hline $\mathrm{t}$ & $3.85 \mathrm{~mm}$ \\
\hline$\beta$ & $13.20^{\prime}$ \\
\hline $\mathrm{d}_{\mathrm{o}}, \mathrm{d}_{\mathrm{i}}$ & $153.5 \mathrm{~mm}, 40.7 \mathrm{~mm}$ \\
\hline $\mathrm{d}_{\mathrm{p}}, \mathrm{d}_{\mathrm{c}}$ & $130.75 \mathrm{~mm}, 40.7 \mathrm{~mm}$ \\
\hline $\mathrm{w}_{1}, \mathrm{w}_{2}$ & $4.96 \mathrm{~mm}, 25.75 \mathrm{~mm}$ \\
\hline $\mathrm{n}$ & $24 \mathrm{EA}$ \\
\hline $\mathrm{E}, \mathrm{v}$ & $2 * 10^{11} \mathrm{~N} / \mathrm{m}^{2}, 0.3$ \\
\hline
\end{tabular}

First, the initial angle $\theta$ was fixed at $13.2^{\circ}$ using MatLAB simulation. Frictional coefficient $\mu$ was set at $0.2,0.4,0.6,0.8$ and 1.0. Then, the change graph of clamp load and release load in accordance with the displacement of diaphragm spring was obtained. Fig. 4 shows the results of simulation. 

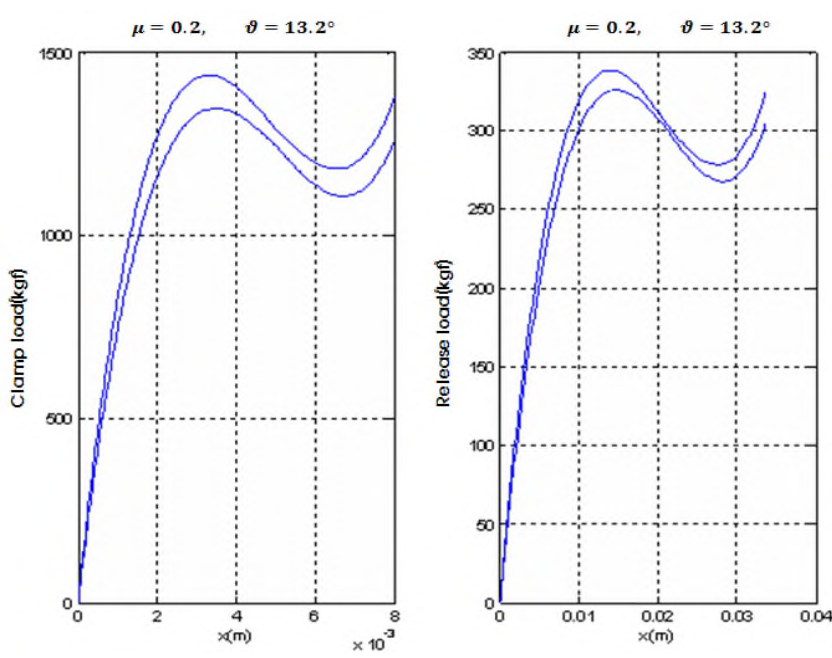

(a) Coefficient of friction $=0.2$

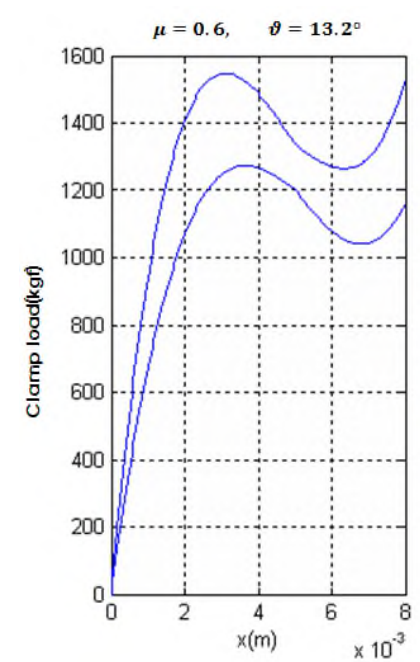

(c) Coefficient of friction $=0.6$
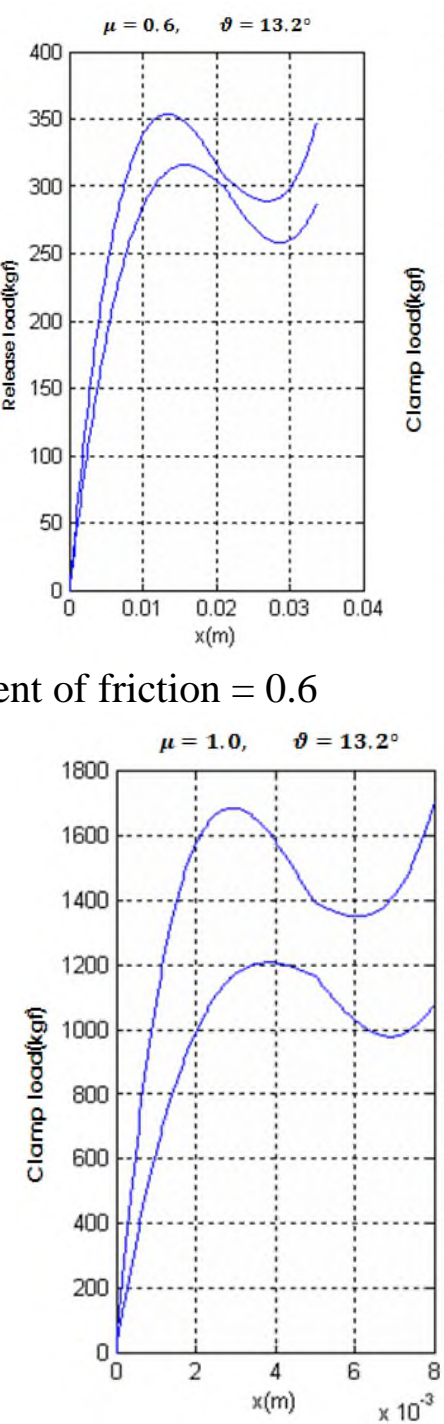
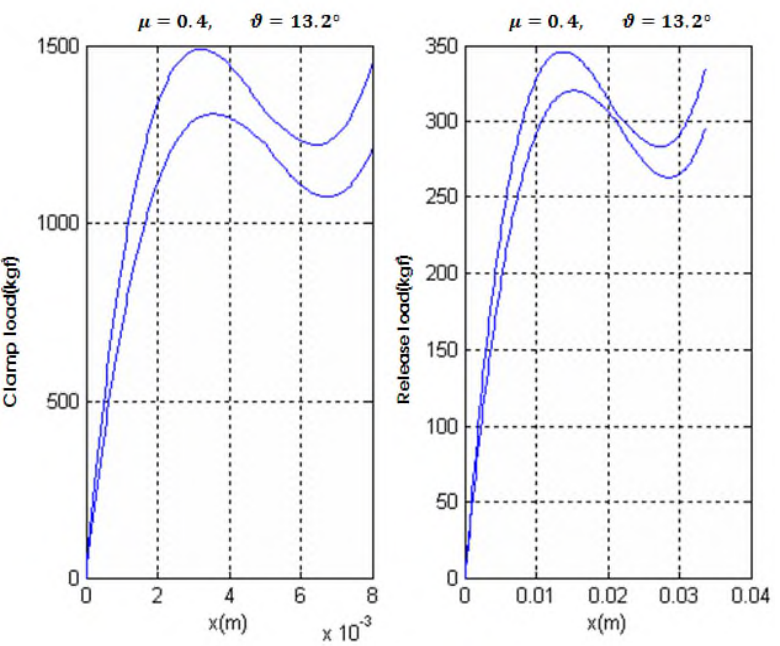

(b) Coefficient of friction $=0.4$
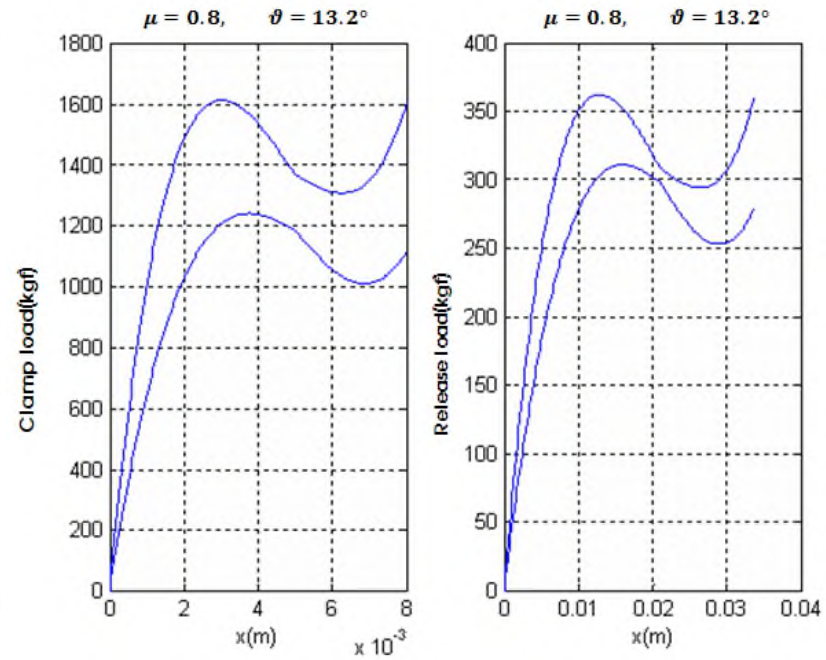

(d) Coefficient of friction $=0.8$

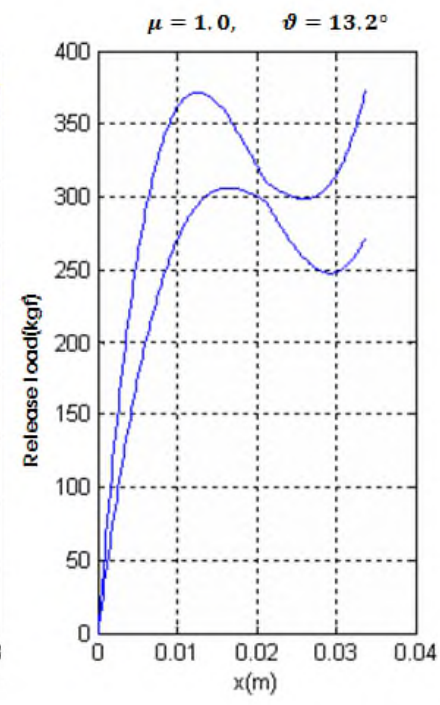

(e) Coefficient of friction $=1.0$

Fig. 3 Results of simulated by variable coefficient of friction (a-e).

As a result of the simulation, the width of hysteresis of displacement-load graph increased in accordance with the value of frictional coefficient. The value used in the existing clutch diaphragm spring is valid when the width of hysteresis curve is $r$ value of approximately $220 \mathrm{kgf}$. The result of the simulation indicates that the most similar result is obtained when frictional coefficient is 0.4 . 


\section{Simulation in Accordance with the Initial Angle}

Next, the characteristics of hysteresis in accordance with displacement-load were analyzed after fixing the frictional coefficient at 0.4 and changing the initial angle to $12,12.5,13,13.5$ and 14 degrees in order to examine the changes in the characteristics based on the changes in the initial angle of diaphragm spring. Fig. 4 shows the results of the simulation.

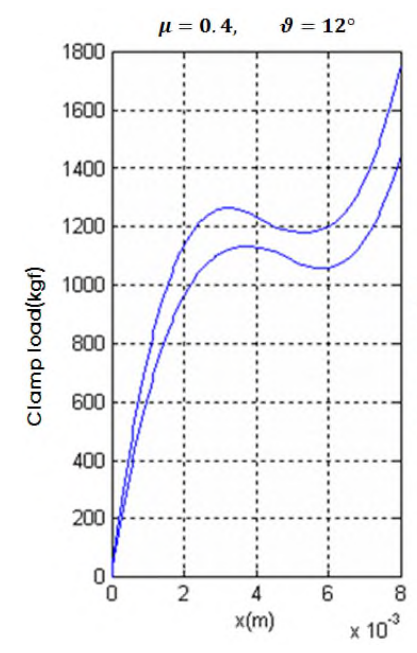

(a) Initial angle $=12$

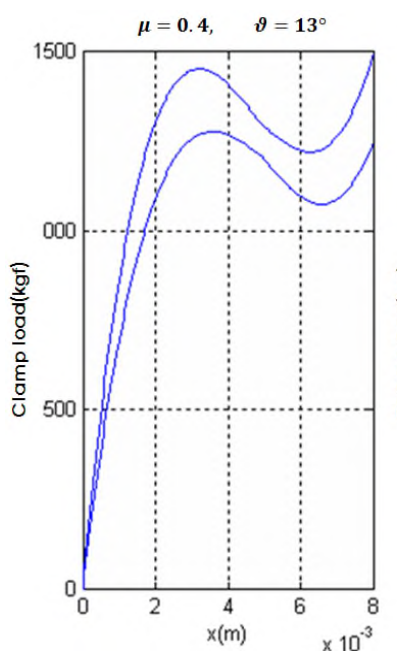

(c) Initial angle $=13$
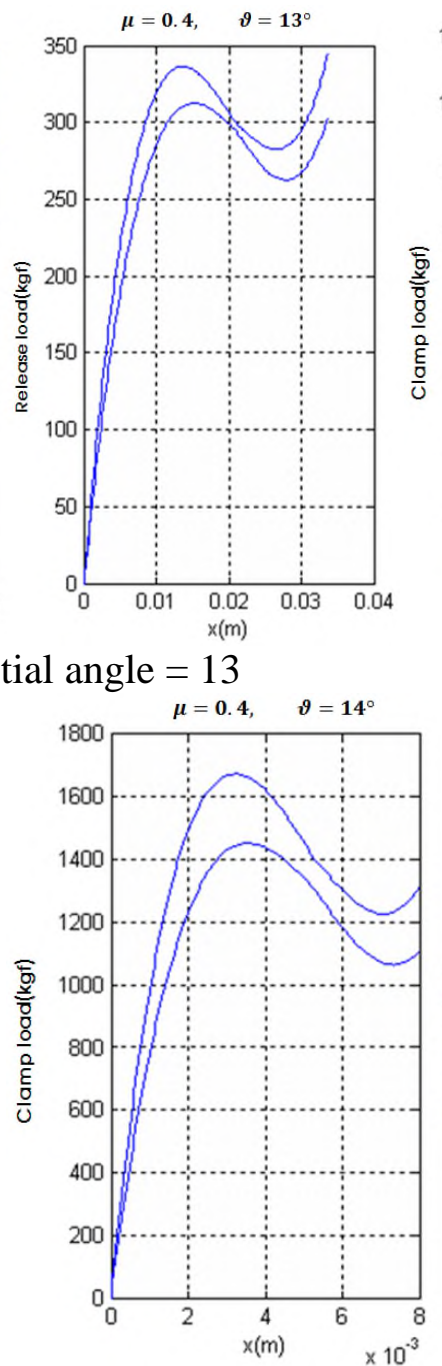
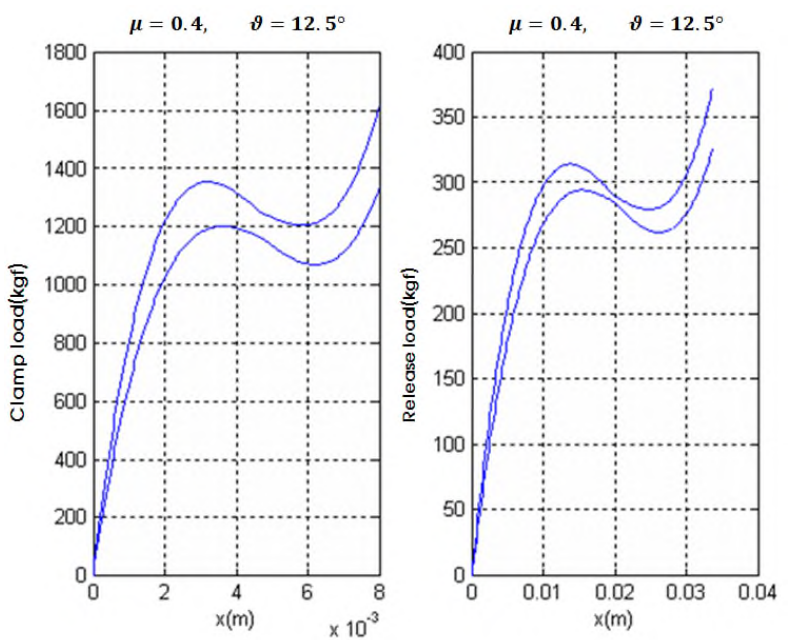

(b) Initial angle $=12.5$
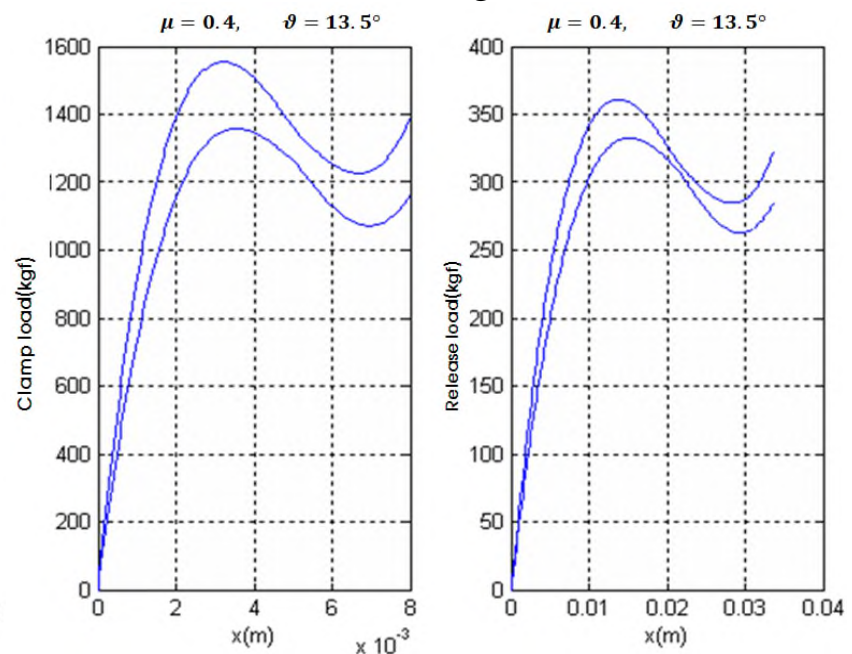

(d) Initial angle $=13.5$

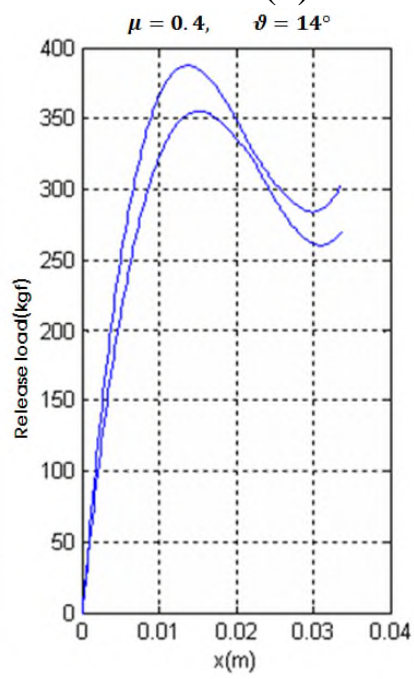

(e) Initial angle $=14$

Fig. 4 Results of simulated by variable initial angle (a-e). 
With the varying initial angle, the shape width ratio of the characteristic curve value of corresponding hysteresis showed a similar movement. However, both clamp load and release load tended to increase with an increase in the angle.

\section{Conclusion}

In this paper, a mathematic model as to the corresponding structure was established and the characteristic changes were analyzed using simulation in order to examine the changes as to clamping load and release load in accordance with the displacement of diaphragm spring of clutch cover in the hydraulic pressure clutch of manual transmission vehicle. In regard to the main variables required for this study in relation to the moment equation, the result values obtained through the existing study were applied to examine the characteristic changes of hysteresis depending on the changes in the frictional coefficient and initial input angle. As a result of the analysis, both clamp load and release load tended to widen the width of hysteresis when the frictional coefficient increased. This result indicates that load also increases along with an increase in frictional force. In addition, the hysteresis value also increased when the initial input angle was increased after making the frictional coefficient constant. This result can be explained by the fact that higher hydraulic pressure is exerted to a clutch system when a driver presses a pedal with a large angle. Comparing it with the widely applied value, a frictional coefficient of 0.4 and an angle of 13 degrees are considered most suitable.

\section{Acknowledgement}

This work was supported by the National Research Foundation of Korea (NRF) Grant funded by the Korean Government (MOE) (No. NRF-2011-0010475).

\section{References}

[1] K. Imai and M. Yamada, Pressure Pulsation Transfer in Clutch Hydraulic System, Toyota central Research \& Development Labs, (1989).

[2] B. S. Lee, Load Characteristics and Sensitivity Analysis for an Automotive Clutch Diaphragm Spring. The Korean Soc. Automot. Eng. 14 (1) (2008) 54-59.

[3] T. Hasebe and H. Yamamoto, Experimental Study of Reduction Methods for clutch Pedal Vibration and Drive Train Rattling Noise from Clutch System, Soc. Automot. Eng. 932007 (1993) 39-44. 